\title{
OsCUL3a-associated molecular switches have functions in cell metabolism, cell death, and disease resistance
}

Zhiqiang Gao ${ }^{1,2,3}$, Qunen Liu ${ }^{1,2}$, Yingxin Zhang ${ }^{1,2}$, Daibo Chen ${ }^{1,2}$, Xiaodeng Zhan ${ }^{1,2}$, Chenwei Deng ${ }^{1,2}$, Shihua Cheng ${ }^{1,2,{ }^{*}}$, Liyong $\mathrm{Cao}^{1,2,{ }^{*}}$

${ }^{1}$ State Key Laboratory of Rice Biology, China National Rice Research Institute, Hangzhou, Zhejiang 310006, China; ${ }^{2}$ Key Laboratory for Zhejiang Super Rice Research, China National Rice Research Institute, Hangzhou, Zhejiang 310006, China; ${ }^{3}$ College of Life Sciences, Gannan Normal University, Ganzhou, Jiangxi 341000, China. *Corresponding authors.

\section{List of Figures legends}

Figure S1. Leaf DAB staining to show ROS buildup in wild-type ZH11, oscul3a mutant, complemented p1300-OsCUL3a-1 and p1300-OsCUL3a-2 at different growth stages.

Figure S2. The protein-protein interaction networks between OsCUL3a interaction partners and identified molecular switch proteins built with STRING analysis.

Figure S3. Comparison of the 1000-grain weight and grain content for two complemented lines: p1300-OsCUL3a-1 and p1300-OsCUL3a-2.

Figure S4. An $O s C U L 3 a$-KO mutant generated by CRISPR-Cas9 has effects similar to an EMS generated oscul3a mutant.

Figure S5. An OsNPR1-KO mutant in the oscul3a mutant background reduces the cell death phenotype in whole plants and leaves, as shown by DAB staining of field grown rice. 
Figure S6. Pathogen infection and leaf phenotype of the oscul3a mutant-related offspring lines in field conditions.

Figure S7. Genotype identification of the oscul3a mutant-related offspring lines.

Figure S8. Pathogen infection phenotypes and leaf DAB staining to assay for ROS vary in oscul3a mutant-related offspring lines.

Figure S9. Rice plant phenotype of the oscul3a mutant and the complemented transgenic seedlings p1300-OsCUL3a-1 and p1300-OsCUL3a-2 grown in field conditions.

\section{List of Tables captions}

Table S1. Sequences of primers used in this study.

Table S2. OsCUL3a-associated molecular switches at only BLM (before lesion mimic formation) stage.

Table S3. OsCUL3a-associated molecular switches at only ALM (after lesion mimic formation) stage.

Table S4. OsCUL3a-associated molecular switches at both BLM- ALM stage.

Table S5. OsCUL3a-associated network is based on STRING analysis.

Table S6. The protein-protein interaction network between OsCUL3a interaction partners and molecular switch proteins identified with STRING analysis.

Table S7. The core protein-protein interaction networks between OsCUL3a interaction partners and molecular switch proteins. 
40 Table S8. OsCUL3a interaction partners, down-regulated and up-regulated molecular switch

41 proteins.

42 Table S9. The interaction between OsCUL3a interaction partners and down-regulated molecular 43 switch proteins.

44 Table S10. The interaction between OsCUL3a interaction partners and up-regulated molecular 45 switch proteins.

46 Table S11. The interaction between down-regulated and up-regulated molecular switch proteins.

47 Table S12. The interaction between OsCUL3a interaction partners and PRM confirmed molecular 48 switch proteins. 

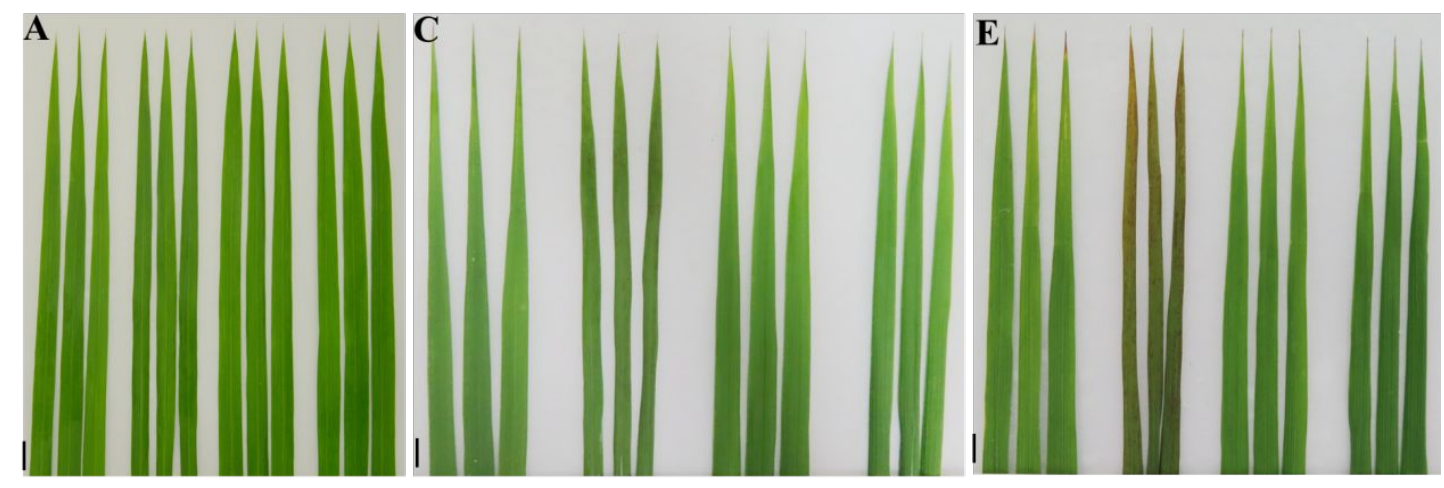

B
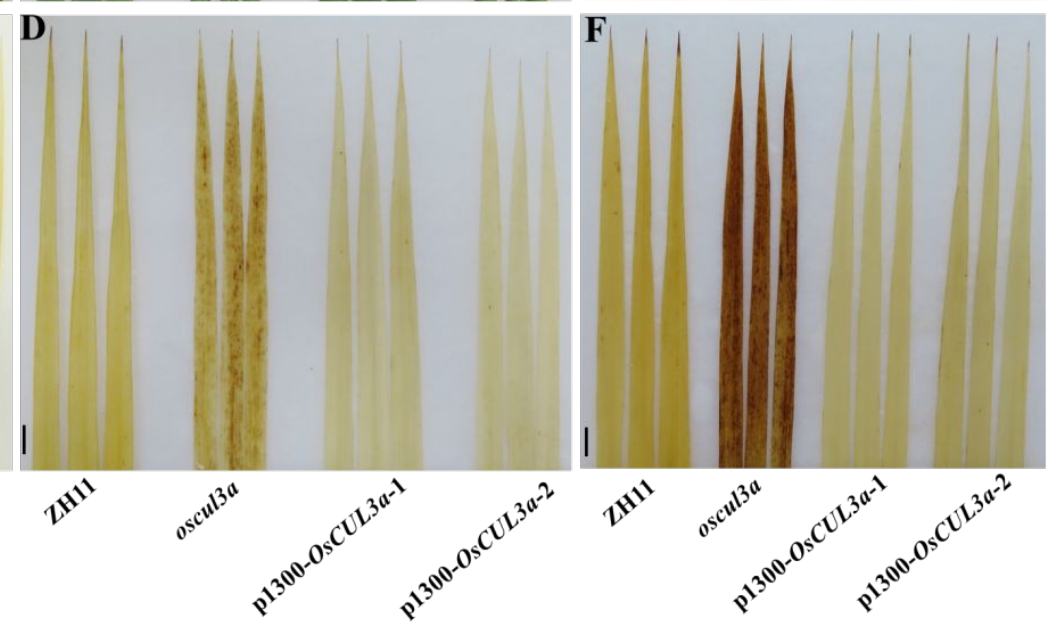

49

50

51

52

53

54

55

Figure S1. Leaf DAB staining to show ROS buildup in wild-type ZH11, oscul3a mutant, complemented p1300-OsCUL3a-1 and p1300-OsCUL3a-2 at different growth stages. (A) and (B), Leaf phenotype (top) and DAB staining (bottom) at 30 DPS. (C) and (D), Leaf phenotype (top) and DAB staining (bottom) at 55 DPS. (E) and (F), Leaf phenotype (top) and DAB staining (bottom) at 70 DPS. Three second top leaves were photographed. Scale bars, $1 \mathrm{~cm}$. DPS, days past sowing. 

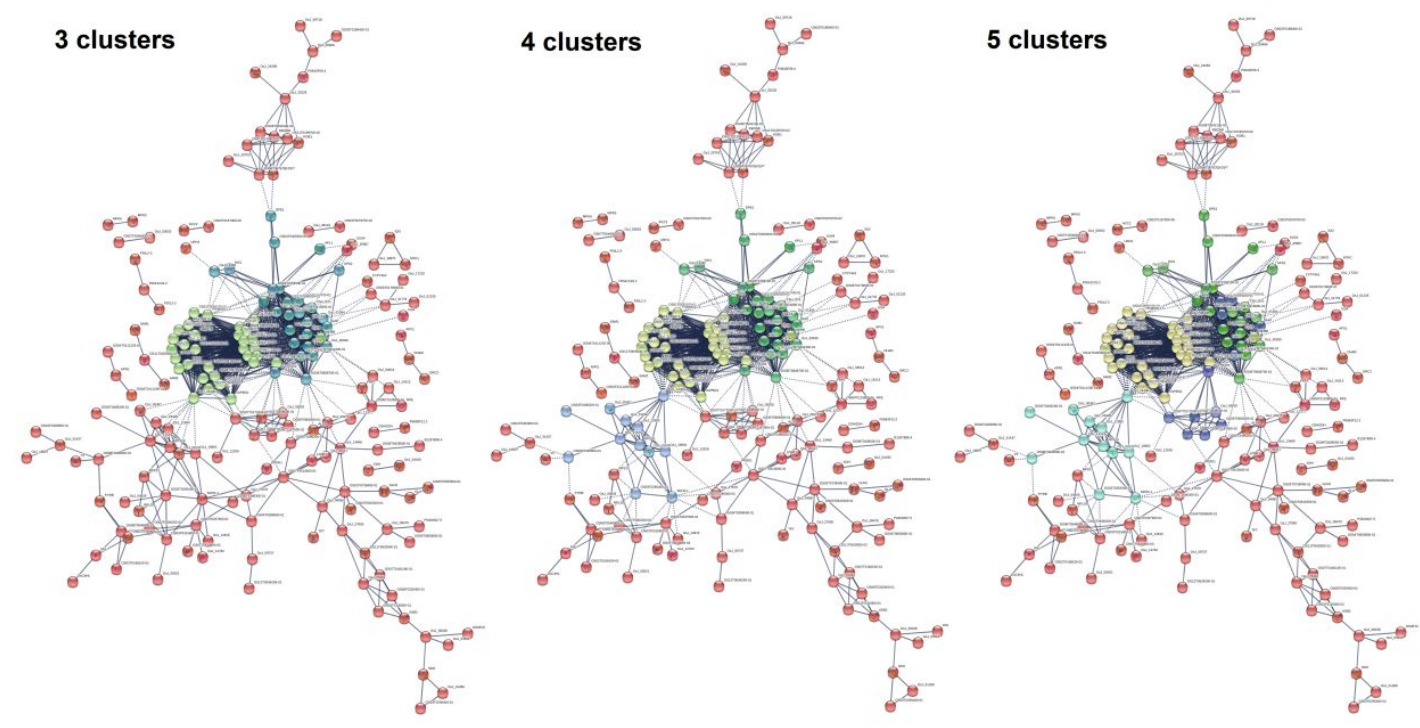

Figure S2. The protein-protein interaction networks between OsCUL3a interaction partners and identified molecular switch proteins built with STRING analysis. We searched the STRING web service using the "protein by name" function for OsCUL3a interaction nodes, and molecular switch proteins were identified from previously obtained proteomic data and using the STRING web service "Multiple proteins" search function. STRING settings used were: meaning of network edges: confidence; minimum required interaction score: medium confidence (0.900); max number of interactors to show: 1st shell (none), 2nd shell (none); network clustering: kmeans clustering method ( 3,4 or 5 clusters). 

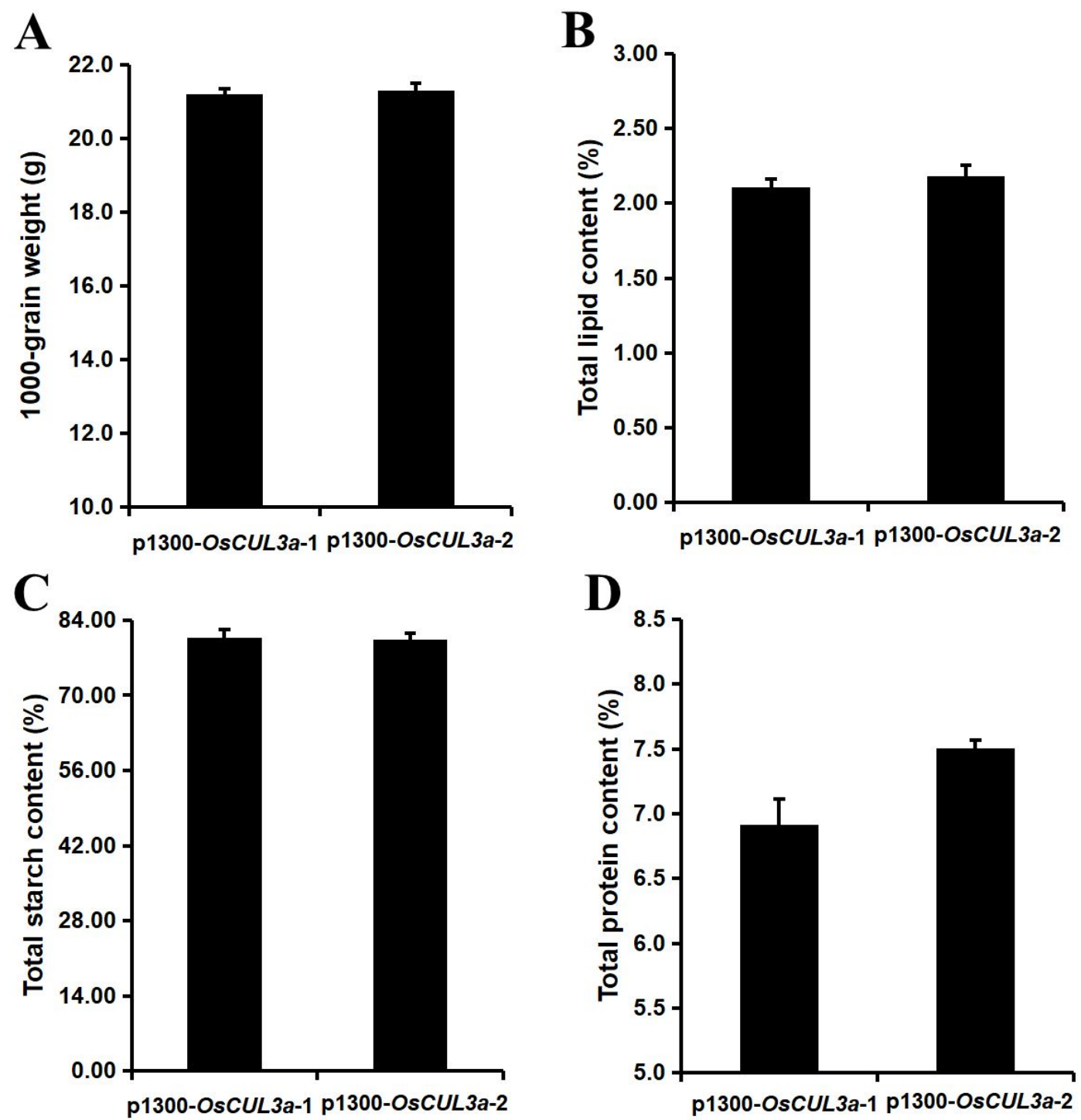

Figure S3. Comparison of the 1000-grain weight and grain content for two complemented lines: $67 \mathrm{p} 1300-O s C U L 3 a-1$ and p1300-OsCUL3a-2. (A), 1000-grain weight of brown rice for 68 p1300-OsCUL3a-1 and p1300-OsCUL3a-2 ( $n=6)$. (B), Total lipid content (\%) of brown rice for 69 p1300-OsCUL3a-1 and p1300-OsCUL3a-2 ( $n=3)$. (C), Total protein content (\%) of brown rice for 70 p1300-OsCUL3a-1 and p1300-OsCUL3a-2 (n=3). (D), Total starch content (\%) of brown rice for $71 \mathrm{p} 1300-O s C U L 3 a-1$ and p1300-OSCUL3a-2 $(n=3)$. Error bars indicate \pm S.D. 

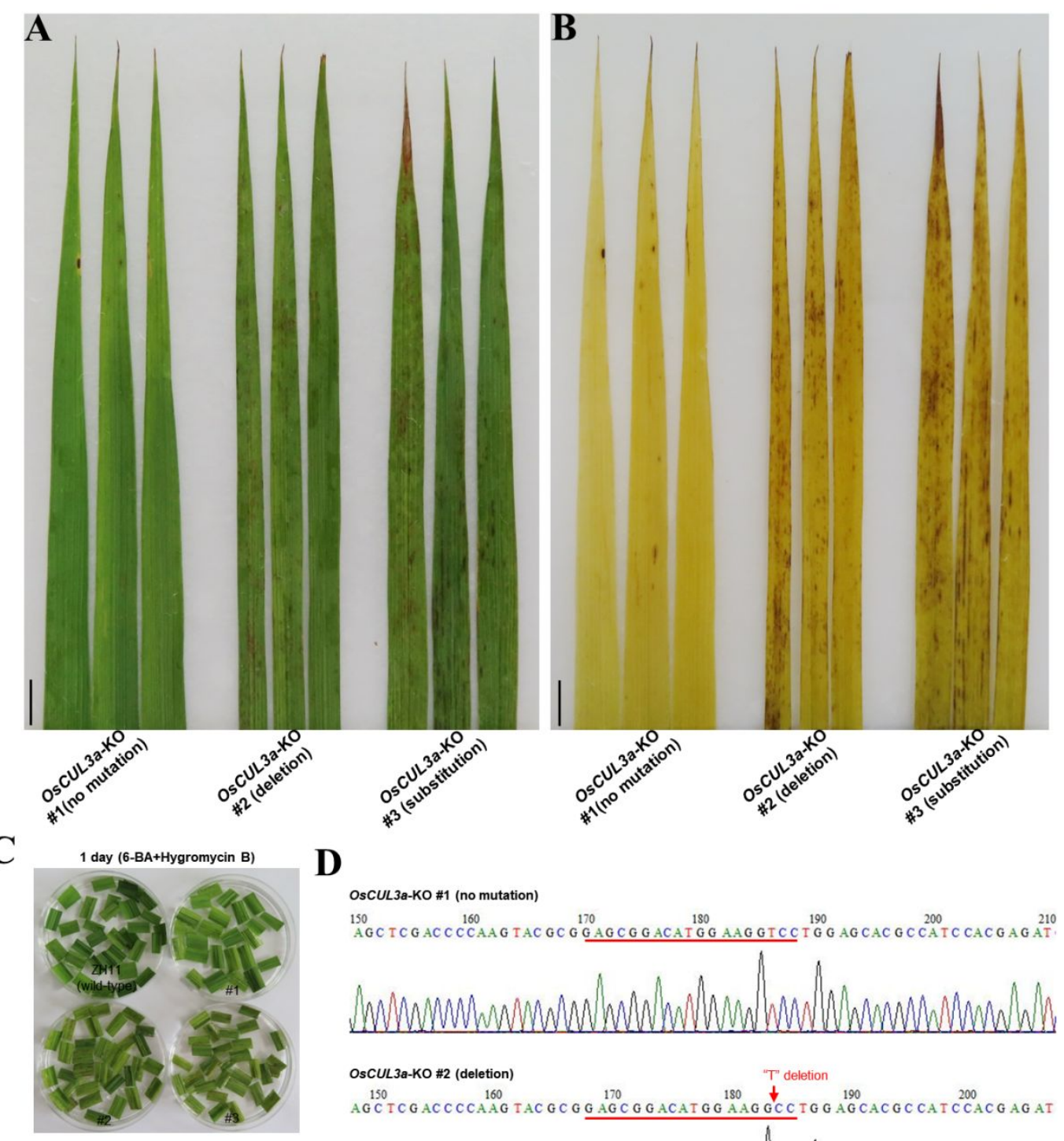

D OsCUL3a-KO \#1 (no mutation)

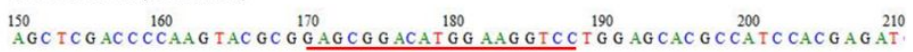

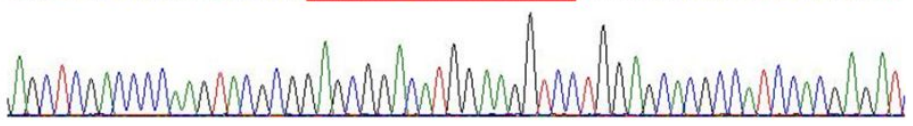

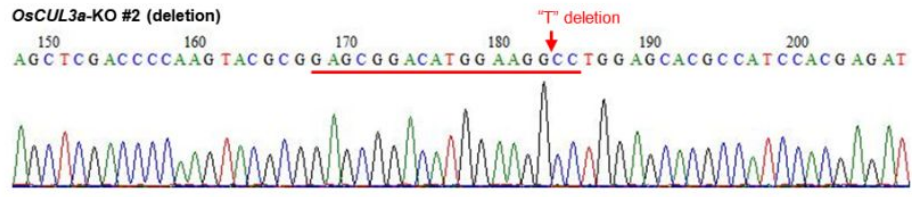
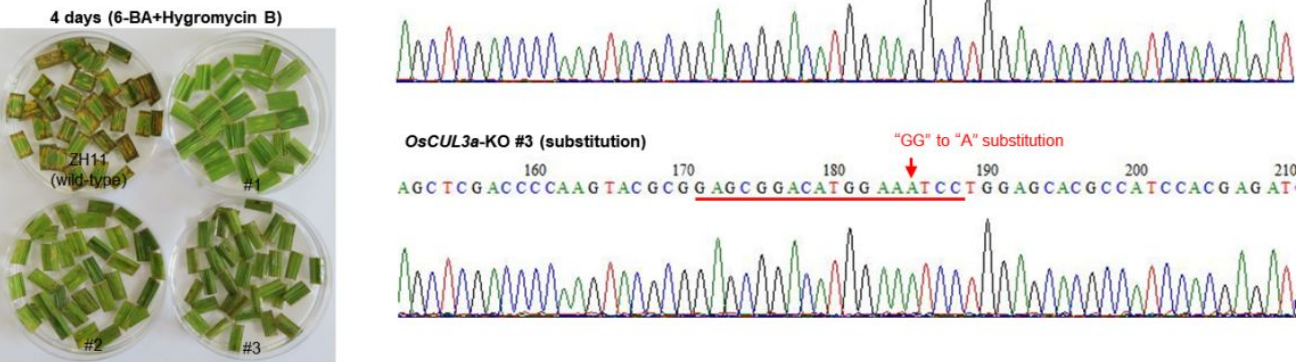

Figure S4. An OsCUL3a-KO mutant generated by CRISPR-Cas9 has effects similar to an EMS generated oscul3a mutant. (A), The leaf phenotype for OsCUL3a-KO \#1 (no mutation), \#2 (deletion), \#3 (substitution) transgenic seedlings in a wild-type ZH11 background. (B), The leaf DAB staining for OsCUL3a-KO \#1 (no mutation), \#2 (deletion), \#3 (substitution) transgenic seedlings in the ZH11 background. (C), The clipped leaves of the wild-type ZH11, OsCUL3a-KO \#1 (no mutation), \#2 (deletion), \#3 (substitution), which were immersed in solution containing $1 \mathrm{mg} / 1$ 6-BA and $25 \mathrm{mg} / \mathrm{l}$ hygromycin B for 4 days treatment. (D), Sequencing confirmation of mutations for different transgenic seedlings. Three second top leaves were photographed. Scale 
bars, $1 \mathrm{~cm}$ for (A) and (B). Red underlines mark CRISPR-Cas9 target sites and the red arrows indicate mutation type and site.
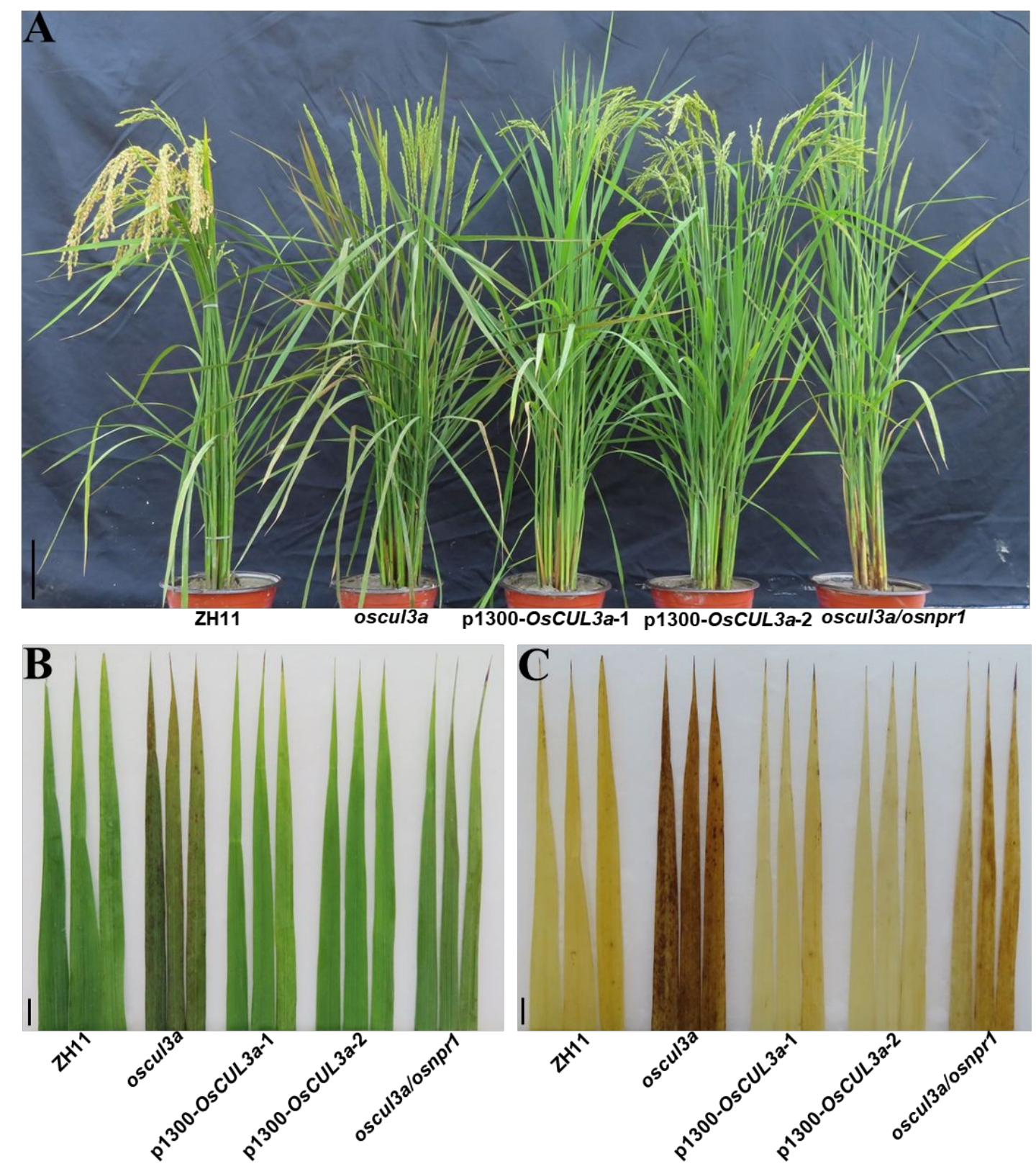

83

84

Figure S5. An OsNPR1-KO mutant in the oscul3a mutant background reduces the cell death phenotype in whole plants and leaves, as shown by DAB staining of field grown rice. (A), The rice plant phenotype of wild-type ZH11, oscul3a mutant, the complemented transgenic seedlings p1300-OsCUL3a-1 and p1300-OsCUL3a-2, and oscul3a/osnpr 1 double mutant grown in the field at 75 DPS. (B), The leaf phenotype for 75 DPS seedlings. (C), The leaf DAB staining for 75 DPS seedlings. The three leaves from left to right are the first top leaf, second top leaf and third top leaf. DPS, days past sowing. Scale bars, $10 \mathrm{~cm}$ for (A), $1 \mathrm{~cm}$ for (B) and (C). 

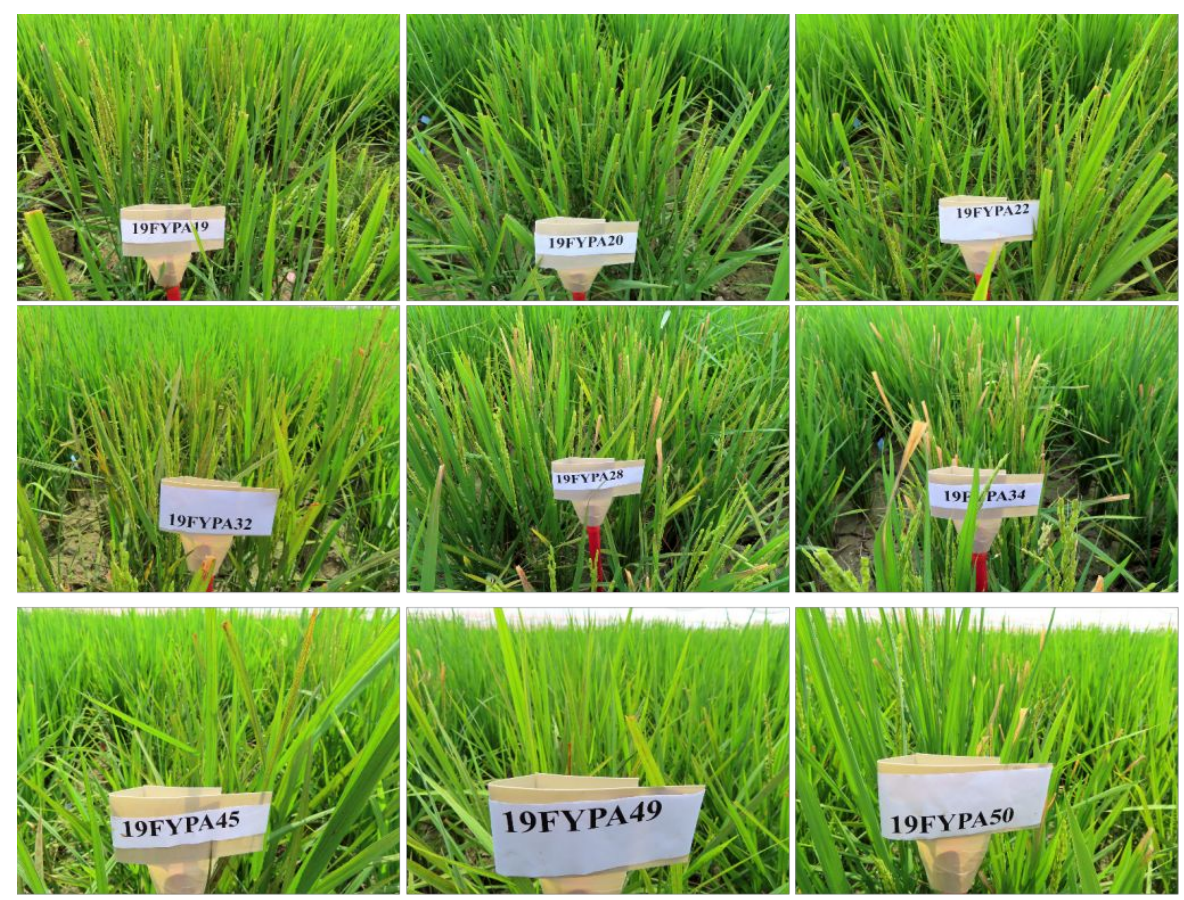

92 Figure S6. Pathogen infection and leaf phenotype of the oscul 3 a mutant-related offspring lines in

93 field conditions. The oscul3a mutant related offspring lines were inoculated with Xoo by the

94 leaf-clipping method at 45 DPS and photographed at 15 dpi (60 DPS). dpi, days past inoculation.

95 DPS, days past sowing. 

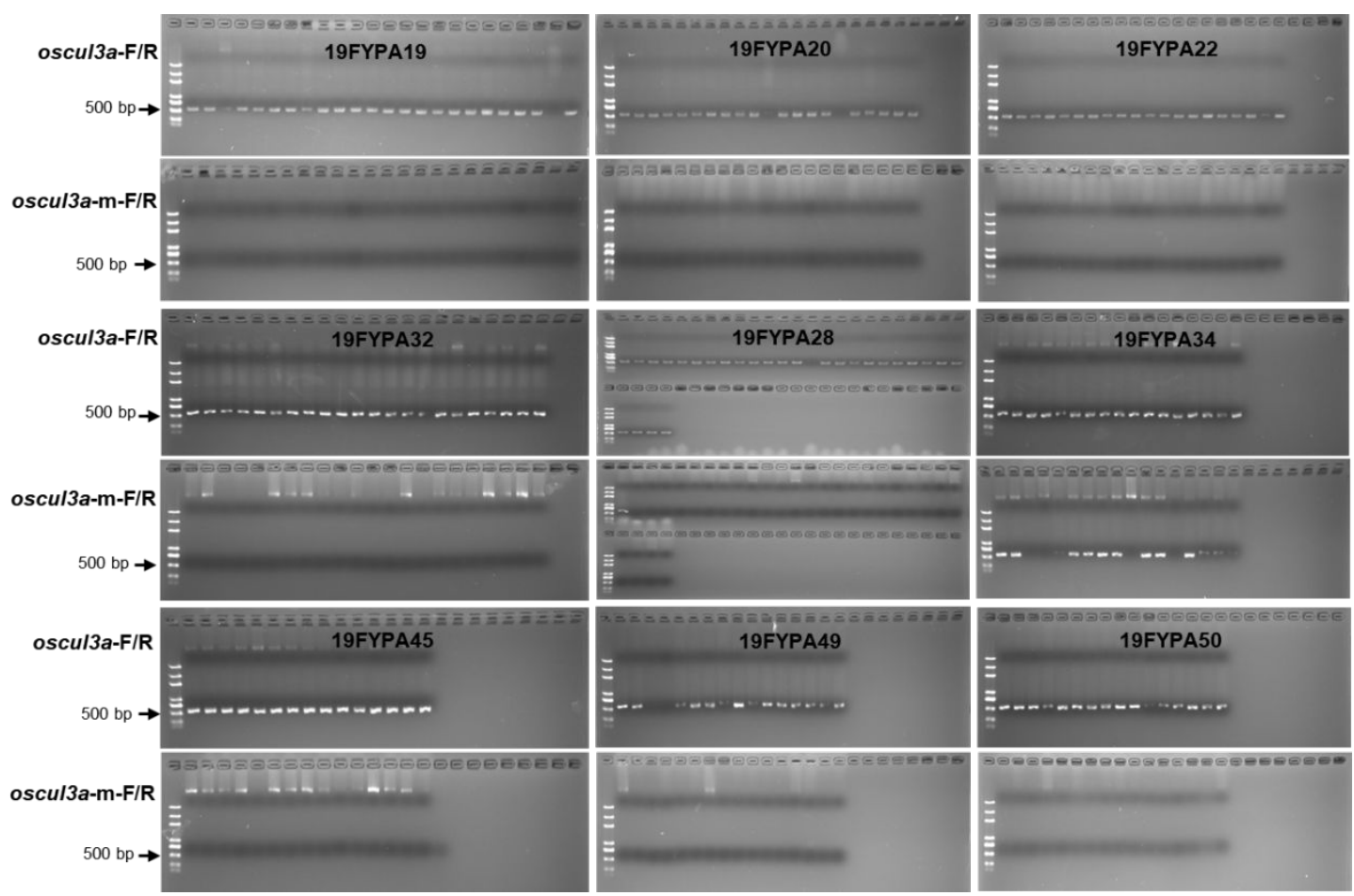

97 Figure S7. Genotype identification of the oscul3a mutant-related offspring lines. The primer oscul $3 a-\mathrm{m}-\mathrm{F} / \mathrm{R}$ is used to confirm the mutant site, with the reverse primer oscul3a-m-R is designed to incorporate the mutated nucleic acid bases. The primer oscul3a-F/R is designed as a positive control to amplify the no mutation sites in the OsCUL3a. The $\sim 500 \mathrm{bp}$ PCR fragment is indicated by arrow. The offspring line 19FYPA34 has no mutation of OsCUL3a as the $\sim 500 \mathrm{bp}$ band after oscul3a-m-F/R amplification. 
A
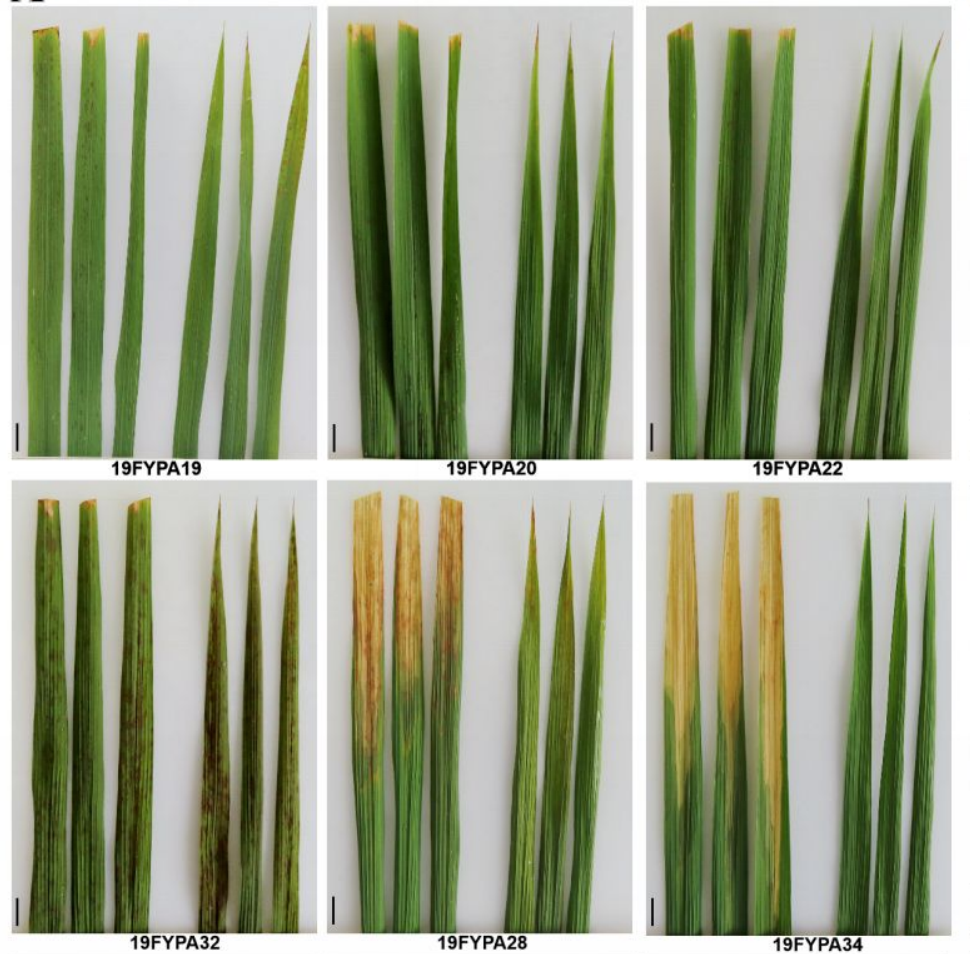
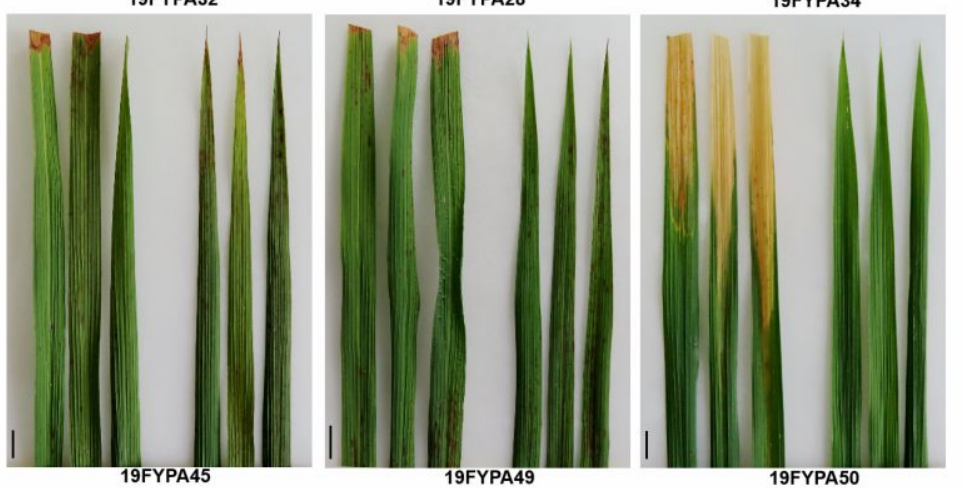

B
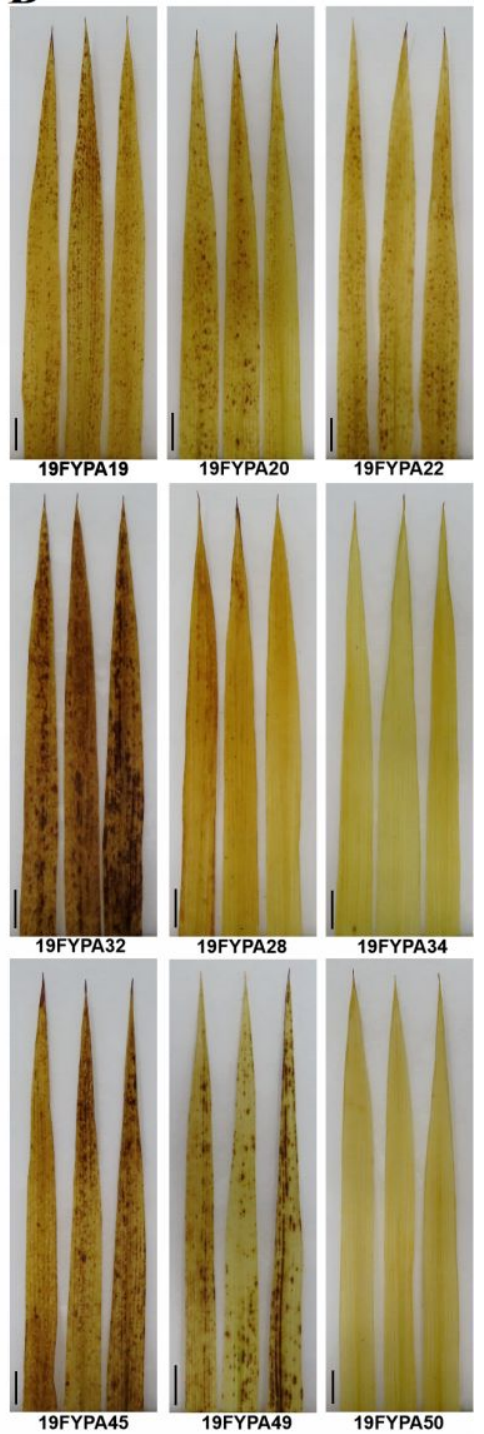

104

Figure S8. Pathogen infection phenotypes and leaf DAB staining to assay for ROS vary in oscul3a mutant-related offspring lines. (A), The oscul3a mutant related offspring lines were inoculated with Xoo by the leaf-clipping method at 45 DPS (photographed at 60 DPS). (B), The leaf DAB staining of the oscul3a mutant related offspring lines at 60 DPS. The top three leaves were photographed for lesion length after pathogen infection in (A), and three uninfected top leaves were also photographed to show intact leaf phenotypes in (A), and then for DAB staining in (B). Scale bars, $1 \mathrm{~cm}$. 

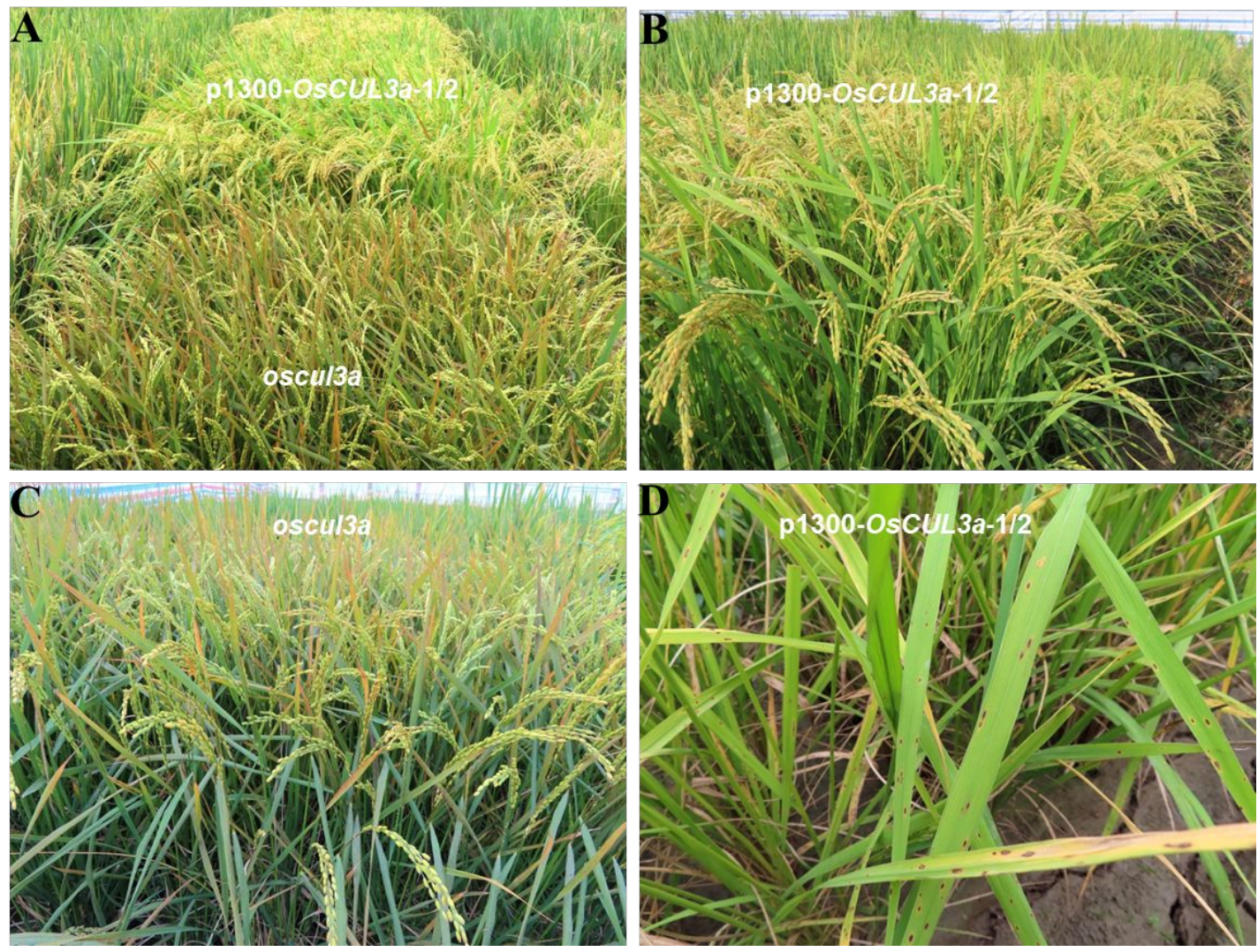

112 Figure S9. Rice plant phenotype of the oscul3a mutant and the complemented transgenic seedlings p1300-OsCUL3a-1 and p1300-OsCUL3a-2 grown in field condition. (A), The visible different yield phenotype of oscul3a mutant and the complemented transgenic seedlings p1300-OsCUL3a-1 and p1300-OsCUL3a-2 (p1300-OsCUL3a-1/2) grown in field condition. (B) and (C), enlarged growth phenotype of p1300-OsCUL3a-1/2 and the oscul3a mutant. (D), The lesions on leaves of p1300-OsCUL3a-1/2 were result of infection by pathogens present in the natural condition. 